\title{
1 Parasite-driven cascades or hydra effects: susceptibility and foraging depression shape
}

\section{2 parasite-host-resource interactions}

3 Jason C. Walsman ${ }^{a^{*}}$, Alexander T. Strauss ${ }^{\mathrm{b}}$, Spencer R. Hall ${ }^{\mathrm{c}}$

4 a University of Pittsburgh ${ }^{b}$ University of Georgia ${ }^{c}$ Indiana University *Corresponding author,

5 walsmanjason@gmail.com

\section{Abstract}

1. When epidemics kill hosts and increase their resources, should the density of hosts decrease (with a resource increase, this constitutes a trophic cascade) or increase (a hydra effect)? Seeking answers, we integrate trait measurements, a resource-host-parasite model, and experimental epidemics with plankton. This combination reveals how a spectrum from cascades to hydra effects can arise. It reflects tension between parasitedriven mortality (a density-mediated effect) and foraging depression upon contact with parasite propagules (a trait-mediated one).

2. In the model, mortality rises when higher susceptibility to infection increases infection prevalence. Epidemics release resources while suppressing hosts (creating a cascade). In contrast, when hosts are less susceptible and parasites depress their foraging, a resource feedback can elevate host density during epidemics (creating a hydra effect), particularly at higher carrying capacity of resources. This combination elevates primary production relative to per-host consumption of resources (two key determinants of host density).

3. We test these predictions of the qualitative effects of host traits and resource carrying capacity with trait measurements and a mesocosm experiment. Trait measurements show clonal lines of zooplankton hosts differ in their foraging depression and susceptibility. We seeded resource-host-parasite mesocosms with different host genotypes and provided 
different nutrient supplies to test model predictions. Hydra effects and trophic cascades arose under different conditions, as predicted by the model.

4. Hence, tension between trait-mediated and density-mediated effects of parasites governs the fate of host density during epidemics - from cascades to hydra effects - via feedbacks with resources.

\section{$\underline{\text { 1. Introduction }}$}

Virulent parasites threaten host populations across taxa (Dobson et al. 2008). Parasites exact fitness costs, increasing mortality and/or reducing fecundity of infected hosts. This fitness harm can decrease host density (Daszak, Cunningham \& Hyatt 2000), potentially contributing to extinction of host populations (Ebert, Lipsitch \& Mangin 2000; Vredenburg et al. 2010). Additionally, harmful outbreaks of parasites can severely damage crops (Fry \& Goodwin 1997) and livestock (Cleaveland, Laurenson \& Taylor 2001; Horan \& Fenichel 2007). Furthermore,

37 epidemics that depress host density can trigger conservation crises, e.g. in mammals (RoelkeParker et al. 1996), birds (Cooper et al. 2009), and amphibians (Vredenburg et al. 2010). consumed by hosts in parasite-driven trophic cascades (Buck \& Ripple 2017). These increased resources may then increase host fitness, making the net effect of parasites on host populations more complex. These net effects matter for community composition (Wood et al. 2007) or biological control (Boivin, Hance \& Brodeur 2012); such concerns emphasize the value of

44 understanding and predicting whether and how strongly parasites will suppress host density and 45 release their resources. 
48 hosts to infection. Since susceptibility promotes infection of new hosts, it typically increases the

49 proportion of hosts infected (Dwyer \& Elkinton 1993; Thrall \& Burdon 2000; Strauss et al.

50 2015; Strauss et al. 2018). Once infected, individual hosts can suffer virulent reductions of

51 fitness such as increased mortality (Ebert, Lipsitch \& Mangin 2000). Multiplied by higher

52 prevalence, such virulence increases population-level mortality and depresses host density

53 (Hochachka \& Dhondt 2000; Hall et al. 2011). Therefore, higher susceptibility should lead to

54 stronger 'trophic cascades', with stronger host suppression and larger resource release. In this

55 sense, susceptibility to parasites acts like the attack rate of predators in predator-prey-resource

56 systems: both modulate mortality of victims and should increase cascade strength (see Appendix:

57 section 1a or Shurin \& Seabloom 2005). Hence, enemy-enhanced mortality can broadly

58 strengthen trophic cascades.

However, natural enemies can also depress their victim's foraging rate. For example,

cues from predators can reduce foraging rates of their prey, e.g., if prey reduce foraging to avoid predation (Morgan 1988; Laundré, Hernández \& Ripple 2010). Similarly, the foraging rate of

62 hosts can slow due to virulent effects of infection, behavioral response to infection, or behavioral 63 avoidance of propagules (Raveh et al. 2011; Hite et al. 2017; Strauss et al. 2019; Hite, Pfenning

$64 \&$ Cressler 2020). Foraging reduction might penalize hosts with lower nutritional intake and

65 fecundity (Buck, Weinstein \& Young 2018). Then again, hosts which eat propagules of parasites might benefit from reduced exposure via foraging reduction (Hite, Pfenning \& Cressler 2020).

67 Additionally, such foraging depression should also indirectly release resources from consumption pressure (Philpott et al. 2004). For hosts that slow their foraging in response to parasites, higher resource density may compensate for slower feeding. Hence, depressed foraging 
71 could be offset by released resources and lower mortality (Morgan 1988; Beckerman, Uriarte \&

72 Schmitz 1997). The net effect of foraging depression in these scenarios is unclear.

Here, we show how the interplay between mortality and foraging depression controls the

74 strength of parasite-driven trophic cascades but can also produce hydra effects. In a hydra effect,

75 a source of mortality (e.g., predator or parasite) leads to higher - not lower - density (e.g. of prey

76 or hosts); one mechanism for this is via a trait-mediated indirect effect on the victim's resources

77 (Matsuda \& Abrams 2004). Resource release can support more or fewer victims, depending on

78 how resource production is divided among victims (Schröder, van Leeuwen \& Cameron 2014).

79 When foraging depression increases production more than per capita consumption of resources, a

80 hydra effect arises via "prudent resource exploitation" (Abrams 2009). Alternatively, if the

81 increase in resource consumption is stronger, then enemies drive a trophic cascade. We apply

82 this theory to parasites that cause both mortality (a density-mediated effect) and foraging

83 depression (a trait-mediated effect). Further, we predict and empirically test how host and

84 resource traits govern the range from hydra effects to strong trophic cascades during epidemics.

Hence, we illustrate how susceptibility, foraging depression, and carrying capacity of the

86 resource lead to both cascades and hydra effects in a single system by interweaving models and

87 experiments. We measured susceptibility and foraging depression traits of several clonal

88 genotypes of zooplankton hosts and used them to parameterize the model. The model predicts

89 that a virulent parasite drives stronger trophic cascades as susceptibility of hosts increases (with

90 or without foraging depression). However, hosts with lower susceptibility but strong foraging

91 depression can experience hydra effects, especially at high carrying capacity of the resource. We

92 tested these predictions using those same host genotypes in a mesocosom experiment. As

93 predicted, populations of more susceptible hosts suffered larger epidemics (higher prevalence) of 
94 a fungal parasite. These larger epidemics more strongly suppressed host density and released

95 algal resources. Further, hydra effects emerged for resistant (low susceptibility) genotypes with

96 strong foraging depression in systems receiving high nutrients. Hence, parasites (like predators)

97 can trigger hydra effects through the "prudent resource exploitation" mechanism. Furthermore,

98

99

100

101

102

103

104

105

106

107

108

109

110

111

112

113

114

115

116

\section{Materials and methods}

\section{(a) Experimental system}

We parameterize and test our model in a planktonic system. We use isoclonal lines of the freshwater zooplankton host (Daphnia dentifera) to manipulate focal traits (Strauss et al. 2015). Hosts incidentally ingest free-living propagules of a virulent fungal parasite (Metschnikowia bicuspidata; Ebert 2005) while filter feeding on algal resources (Ankistrodesmus falcatus). Infected hosts have elevated death rate, and they release infectious propagules upon death. The short generation times of hosts, resources, and parasites enable multi-generation feedbacks during experimental epidemics.

\section{(b) Estimation of susceptibility and foraging traits}

Susceptibility - the probability a host becomes infected given a single exposure modulates population-level mortality from disease. For each of three clonal genotypes, we estimated susceptibility from the number of hosts becoming infected following controlled time and dose of propagules (Strauss et al. 2015). For these same host genotypes, we quantified depression of foraging rate when hosts contact spore propagules. Foraging rate of individual 
117 hosts was measured for eight hours at different spore concentrations (Strauss et al. 2019). Since

118 terminal infection develops over longer time ( $\sim 9$ days, Stewart Merrill et al. 2019$)$, the measured

119 depression of foraging rate reflects behavior more than a symptom of infection. We fit foraging

120 data for genotypes 1 and 2 (by Strauss et al. 2019, re-analyzed here) and for genotype 3

121 (unpublished) as an exponential function of spores $f(Z)=f_{0} \mathrm{e}^{-\alpha Z}$ (where $f_{0}$ is the maximum

122 foraging rate; see Appendix: section 2a). We determined significance of differences in these

123 coefficients of foraging depression $(\alpha)$ by bootstrapping 95\% CIs (Efron \& Tibshirani 1993 see

124 Appendix: section 2a for details). All analyses were performed in Rstudio (R Core Team 2019).

125

\section{(c) Resource-host-parasite model}

We analyze a minimal model of logistically growing resources $(R)$, susceptible hosts $(S)$, biology of focal planktonic system (see also Table 1):

$$
\text { Resources: } \frac{d R}{d t}=r R\left(1-\frac{R}{K}\right)-f(Z)(S+I) R
$$

$$
\text { Susceptible hosts: } \frac{d S}{d t}=c f(Z)(S+I) R-d S-u f(Z) S Z
$$

$$
\text { Infected hosts: } \frac{d I}{d t}=u f(Z) S Z-(d+v) I
$$

$$
\text { Spores: } \frac{d Z}{d t}=\sigma(d+v) I-m Z
$$

Resources grow logistically, a reasonable assumption allowing primary productivity to

135 (potentially) increase during epidemics. Primary productivity is population-level recruitment of new resources per unit time: $r R(1-R / K)$. Resource growth and production depend on intrinsic rate

137 of increase $r$ and carrying capacity $K$ (first term, eq. 1a). Resources are consumed by susceptible 
139

140

141

142

143

144

145

146

147

148

149

150

151

152

153

154

155

156

157

158

159

160

161

exponentially with density of spores $(Z)$; this exponential function prevents the unrealistic case of a negative feeding rate at high $Z$. This trait-mediated effect of parasites and may be strong (large $\alpha$ ) or absent $(\alpha=0)$. We assume equal foraging rate and foraging depression for susceptible and infected classes as a first, simplest approach (see Penczykowski et al. 2020 for a reduced foraging rate by infected hosts). Both host classes, $S$ and $I$, convert resources into susceptible offspring (i.e., horizontal, environmental transmission; first term, eq. 1b; see Appendix: section $1 \mathrm{~b}$ for reduced fecundity of infected hosts) with conversion efficiency $c$. For simplicity, infection does not lower fecundity. Total host density is $H=S+I$. Hosts experience background mortality at rate $d$ (second term, eq. 1b) due to predation, senescence, and other factors. Susceptible hosts encounter parasites while foraging; their susceptibility, $u$, determines the proportion of hosts infected per encounter. Exposure and susceptibility jointly determine the transmission rate (often denoted $\beta$ ) from the environment to susceptible hosts, $u f_{0} e^{-\alpha Z}$ (third term, eq. 1b). Infection converts susceptible hosts into infected hosts (first term, eq. 1c). These hosts suffer elevated death rate due to virulence of infection, $d+v$ (where $v$ is pathogen-induced mortality; second term eq. 1c). Death of infected hosts is the density-mediated effect of parasites. When infected hosts die, they release $\sigma$ parasite propagules $(Z)$ back into the environment (first term, eq. 1d). Losses of parasite propagules occur at background rate $m$ (second term, eq. 1d), e.g., due to sinking, consumption (Strauss et al. 2015), solar radiation (Overholt et al. 2012), etc. The model has a single, stable, endemic equilibrium for the range of parameter values considered. Since the exponential form of $f_{0} e^{-\alpha Z}$ prevented general analytical solutions (though see eq. 2 for partial solutions), we found equilibrial densities at each parameter set with a root finder and evaluated stability of each using local stability analysis in Rstudio version 3.6.0 (R Core Team 2019). We then determine the effects of prevalence $(p)$, susceptibility $(u)$, foraging 
162 depression $(\alpha)$, and carrying capacity $(K)$ on resource $(R)$ and host $(H)$ densities using a mixture

163 of analytical and numerical techniques. We defined the strength of trophic cascades (resource

164 release with host suppression) or hydra effects (resource release with host increase) as ratios of

165 the stable endemic equilibrium to the disease-free boundary equilibrium. Since the mesocosm

166 epidemics likely did not reach equilibria, we compared the closet analogue: we qualitatively

167 matched them to transient dynamics of the model, and use the equilibria of the model to predict

168 longer term outcomes. (See Appendix: section 1c for parameter values in which oscillations or

169 bistability [when $\alpha>0$ ] arise).

170 Table 1. Symbols for state variables (top) and traits and other parameters (bottom) in the

171 dynamical model (eq. 1). Default values are accompanied by ranges.

\begin{tabular}{|c|c|c|c|}
\hline Symbol & Meaning & Units & Value \\
\hline$t$ & Time & day & Varies \\
\hline$R$ & Density of resources & $\mu \mathrm{g} \operatorname{chl} a \cdot \mathrm{L}^{-1}$ & Varies \\
\hline$S$ & Density of susceptible hosts & hosts $\cdot L^{-1}$ & Varies \\
\hline$I$ & Density of infected hosts & hosts $\cdot \mathrm{L}^{-1}$ & Varies \\
\hline$H$ & Total host density, $S+I$ & hosts $\cdot \mathrm{L}^{-1}$ & Varies \\
\hline$Z$ & Density of parasite propagules & parasites $\cdot \mathrm{L}^{-1}$ & Varies \\
\hline$p$ & Prevalence of infection, $\frac{I}{S+I}$ & unitless & Varies \\
\hline$c$ & Conversion efficiency, hosts & hosts $\mu \mathrm{g} \operatorname{chl} a^{-1}$ & $0.18^{*}$ \\
\hline$d$ & Background mortality rate & day $^{-1}$ & $0.011^{*}$ \\
\hline$f_{0}$ & Foraging rate, maximum & $\mathrm{L} \cdot$ host $^{-1} \cdot$ day $^{-1}$ & $0.0138^{+}$ \\
\hline
\end{tabular}




\begin{tabular}{|c|c|c|c|}
\hline$f(Z)=f_{0} \mathrm{e}^{-\alpha Z}$ & Foraging rate, function of $Z$ & $\mathrm{~L} \cdot$ host $^{-1} \cdot$ day $^{-1}$ & See Fig. 1 \\
\hline$K$ & Carrying capacity, resources & $\mu \mathrm{g} \operatorname{chl} a \cdot \mathrm{L}^{-1}$ & $94.3 ; 10-100^{*}$ \\
\hline$m$ & Loss rate of parasites & day $^{-1}$ & $1.93^{\dagger}$ \\
\hline$r$ & Intrinsic rate of increase, $R$ & day $^{-1}$ & $0.52^{\ddagger}$ \\
\hline$u$ & Susceptibility to parasites & host $\cdot$ parasite $^{-1}$ & $5.81 \times 10^{-5} ; 0.157-4.35 \times 10^{-4 *}$ \\
\hline$v$ & Pathogen-induced mortality & day $^{-1}$ & $0.045 ; 1.05 \times 10^{-5}-0.06^{*}$ \\
\hline$\alpha$ & Foraging depression, hosts & $\mathrm{L} \cdot$ parasite $^{-1}$ & $3.45 \times 10^{-6} ; 0-2.27 \times 10^{-5 \dagger}$ \\
\hline$\sigma$ & Parasite production per host & parasites $\cdot$ host $^{-1}$ & $1.32 \times 10^{5^{*}}$ \\
\hline
\end{tabular}

\section{(d) Mesocosm test of model predictions}

We test predictions of the dynamic model with populations of interacting algal resources,

175

176

177

178

179

180

181

182

183

184

185

186

${ }^{*}$ Biologically reasonable (fm. Strauss et al. 2015) ${ }^{\dagger}$ Reasonable given data ${ }^{\star}$ A reasonable estimate 
187 or absent; each population treatment was replicated three times; 72 total mesocosms). With twice

188

189

190

191

192

193

194

195

196

197

198

199

200

201

202

203

204

205

206

207

208

209

weekly sampling, we measured algal density, host density, and infection prevalence over c.

seven host generations ( $\sim 1$ generation every 10 days; see Appendix: sections $2 \mathrm{~b}, \mathrm{c})$.

We assume that temporal averages approximate equilibria from the model. In that model, connections between susceptibility $(u)$ and prevalence $(p)$ prove important mathematically.

Hence, we first tested for their relationship (and with nutrient supply) using beta regression (see Appendix: section $2 \mathrm{~d}$ ). We next $\log _{10}$-transformed algal and host density, respectively, to

disease, susceptibility, and nutrients. Log ratios reduce bias while having more normal error distributions (Hedges, Gurevitch \& Curtis 1999). When disease drives a trophic cascade, it should have a significant positive effect on resource density and negative effect on host density.

If susceptibility increases trophic cascade strength, there should be a significant positive interaction between susceptibility and disease for $\log _{10}$ resource density and negative interaction for $\log _{10}$ host density (see Appendix: section 2e). Additionally, we tested for a hydra effect in two genotype treatments at high nutrient supply using a nested ANOVA (see Appendix: section $2 \mathrm{f})$. For each genotype, $\log _{10}$ host density was the response variable with mesocosms nested within disease treatment and repeated measurements nested within mesocosms. This nesting incorporated the power and autocorrelation of repeated measurements. Out of the original 72 mesocosms, nine were removed as outliers (most due to population extinction; see Appendix: section 2c). All analyses were performed in Rstudio (R Core Team 2019).

\section{$\underline{\text { 3. Results }}$}

(b) Estimation of susceptibility and foraging traits

The range of trait values occupied by the three host genotypes facilitates manipulation of 
210

211

212

213

214

215

216

217

218

219

population-average traits. The three genotypes span almost an order of magnitude of susceptibility (Bristol 10: $u_{1}=5.81 \times 10^{-5}, \mathrm{~A} 4-3: u_{2}=1.09 \times 10^{-4}$, Standard: $u_{3}=3.93 \times 10^{-4}$ ). All host genotypes significantly depressed their foraging in the presence of parasites (decreasing curves in Fig. 1a; coefficients of foraging depression $[\alpha]$ with 95\% CIs not overlapping zero; Fig. 1b). Foraging depression was more than twice as strong for genotype $1\left(\alpha=3.5 \times 10^{-7} \mathrm{~L} \mathrm{spore}^{-1}\right)$ as for genotypes 2 or $3\left(1.4 \times 10^{-7}\right.$ and $7.6 \times 10^{-8}$, respectively; 95\% CIs of genotype 1 do not overlap those of the other two; Fig. 1b). Given this coefficient of foraging depression, the foraging rate of genotype 1 decreased by a factor of four over the range of spore doses in the trait assay (roughly corresponding to range of spore densities in the mesocosm, see Fig. S1).
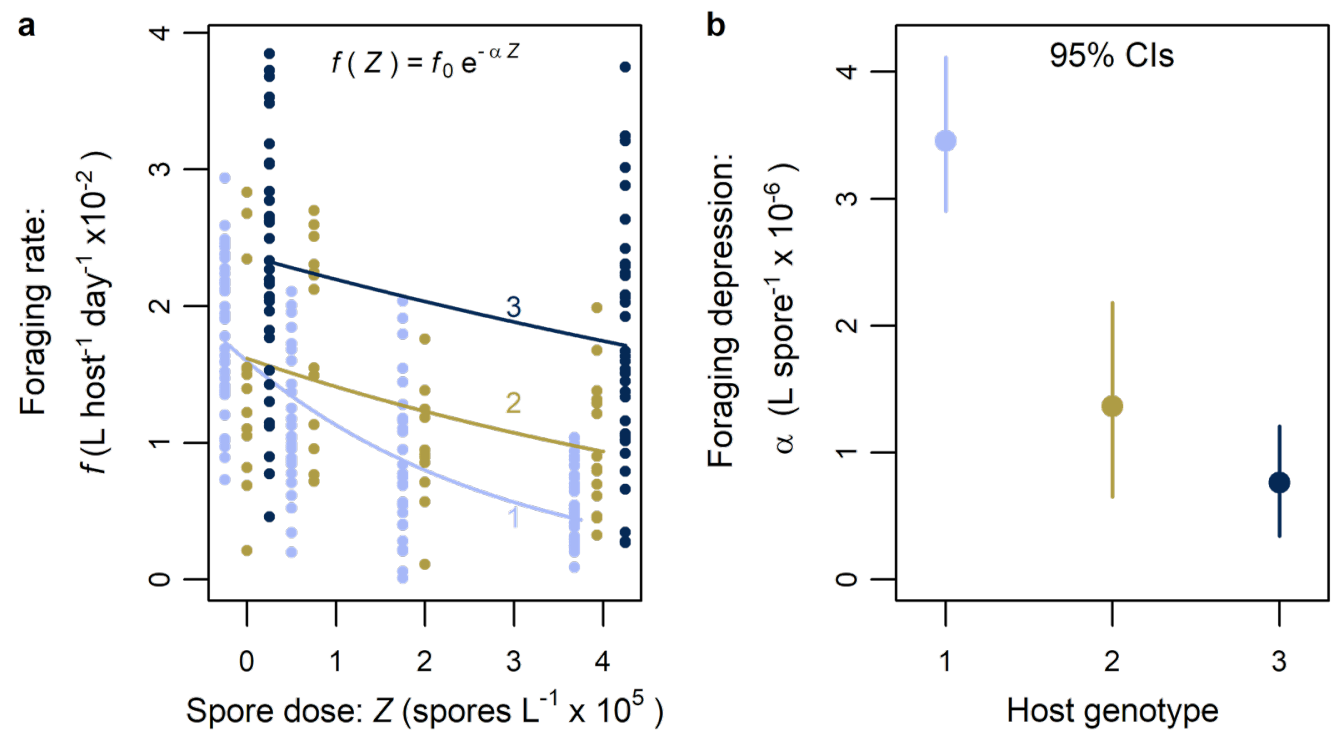

Figure 1. Differences among genotypes in the coefficient of foraging depression ( $\alpha$ ). (a) Fits of an exponential decay model for foraging rate with spores, $f(Z)=f_{0} \mathrm{e}^{(-\alpha Z)}$ to data from a foraging experiment. Larger values of $\alpha$ indicate stronger depression of foraging rate with spore dose, $Z$. Points are jittered horizontally for clarity. (b) The coefficient of foraging depression $(\alpha)$ for genotype 1 was stronger than for other two genotypes (2 and 3). Error bars are boot-strapped 


\section{(c) Resource-host-parasite model}

229

230

231

232

233

234

235

236

237

238

239

240

241

242

Susceptibility modulates host mortality while foraging depression $(\alpha>0)$ enables the parasite-driven hydra effect. When host populations are more susceptible, they suffer higher prevalence of infection and thus higher mortality. When parasites drive higher mortality, they release resources and suppress host density more, producing a stronger trophic cascade. Hence, susceptibility acts analogously to attack rate of predators (see Appendix: section 1a and Shurin \& Seabloom 2005). Yet, with lower susceptibility and mortality, foraging depression can drive a hydra effect. Insight arises from the numerator and denominator of host density (Schröder, van Leeuwen \& Cameron 2014) derived from the resource equation (eq. 1a). Host density $\left(H^{*}=\right.$ $\left.S^{*}+I^{*}\right)$ is the ratio of primary productivity, $P P=r R^{*}\left(1-R^{*} / K\right)$, to per host food consumption, $F C=$ $f\left(Z^{*}\right) R^{*}$. Resource density, $R^{*}$, in turn, is host death rate $\left[d+v p^{*}\right.$, with prevalence $\left.p=I^{*} / H^{*}\right]$ divided by fecundity per resource available $\left[c f\left(Z^{*}\right)\right]$. Thus, host density depends on productivity and consumption of resources:

$$
\text { Resources without disease: } R_{Z-}^{*}=\frac{d}{c f_{0}}
$$

$$
\text { Resources with disease: } R_{Z+}^{*}=\frac{d+v p^{*}}{c f\left(Z^{*}\right)}
$$

$$
\text { Hosts without disease: } H_{Z-}^{*}=\frac{P P_{Z-}}{F C_{Z_{-}}}=\frac{r R_{Z-}^{*}\left(1-R_{Z-}^{*} / K\right)}{f_{0} R_{Z-}^{*}}
$$

$$
\text { Hosts with disease: } H_{Z+}^{*}=\frac{P P_{Z+}}{F C_{Z+}}=\frac{r R_{Z+}^{*}\left(1-R_{Z+}^{*} / K\right)}{f\left(Z^{*}\right) R_{Z+}^{*}}
$$

Because disease raises mortality, it still must increase the minimal resource requirement of hosts (compare eqs. $2 \mathrm{a}, \mathrm{b} ; R^{*}{ }_{Z^{+}}>R^{*}{ }_{Z-}$ since $f(Z) \leq f_{0}$ and $d+v p^{*}>d$ ). Released resources increase or decrease primary productivity by drawing nearer to or farther from $K / 2$ (because $P P$ is 
248

249

250

251

252

maximized at $K / 2) . P P$ drops when hosts had high minimal requirements without disease $\left(R^{*} Z_{-}>\right.$ $K / 2$ ). Lower $P P$, in turn, necessarily decreases host density (permitting only a cascade). But if hosts more strongly controlled resources without disease $\left(R^{*} Z_{-}<K / 2\right)$, resource release can increase $P P$ (if $R^{*}{ }_{Z+}$ is closer to $K / 2$ than $R^{*}$-). However, increased $P P$ alone may not suffice: hydra effects only emerge when increased $P P\left(P P_{Z^{+}}>P P_{\mathrm{Z}-}\right)$ outweighs increased food consumption per host $\left(F C_{Z^{+}}>F C z\right.$; eq. $\left.2 \mathrm{c}, \mathrm{d}\right)$.

Hence, food consumption places an additional constraint on the hydra effect. Food consumption per host $(F C)$ is the product of foraging rate, $f\left(Z^{*}\right)$, and the density of resources, $R^{*}$. Higher mortality from infection increases both $R^{*}$ and $F C$ when foraging rates stay constant $[\alpha=$ 0 , so $f\left(Z^{*}\right)=f_{0}$ ]. If parasites only kill hosts $(v>0, \alpha=0), F C$ increases more with $R^{*}$ than primary productivity can [since $H^{*} Z^{+}$simplifies to $r\left(1-R^{*} / K\right) / f_{0}$, a decreasing function of $R^{*}$ ]. So, parasites that only kill must decrease host density. If parasites only depress host foraging rate $(v$ $=0, \alpha>0)$, resource density with disease simplifies to $R^{*}{ }_{Z^{+}}=d /\left[c f\left(Z^{*}\right)\right]$ (from eq. 2b). This minimal resource requirement, $R^{*}{ }^{+}$, compensates for foraging depression completely; hence, $F C$

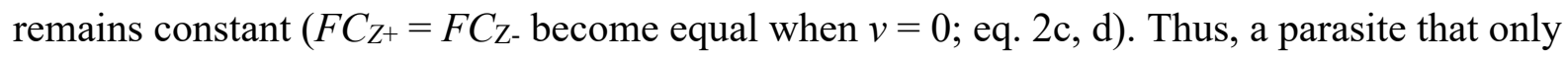
depresses foraging rate of hosts (but does not kill) will increase host density if primary productivity increases with disease. If parasites increase mortality and depress host foraging rate $(v>0, \alpha>0)$, they can drive a hydra effect if the increase in $P P$ outweighs the simultaneous increase in $F C$ per host. Thus, a tension between foraging depression and mortality emerges because they differentially influence production and consumption of resources.

These dueling effects of production and consumption explain why hydra effects become more likely with higher carrying capacity of the resource $(K)$. Higher $K$ elevates density of parasite propagules in the environment $(Z)$ in both the mortality-only case $(v>0, \alpha=0$; dashed 
271 red) and the foraging-depression case ( $v>0, \alpha>0$; solid red curve; Fig. 2a). More propagules, in

272 turn, decrease foraging rate (when $\alpha>0$; Fig. 2b). Resources $\left(R^{*}\right)$ also increase with carrying

273 capacity, but more so if hosts depress foraging (Fig. 2c). That larger release of resources can

274 create a stronger increase in primary productivity - and more so at higher $K$ (Fig. $2 \mathrm{~d})$. At the

275 same time, food consumption per host (Fig. 2e) increases with $K$ but less so with foraging

276 depression than without. Hence, epidemics more easily drive a hydra effect at higher $K$ (Fig. 2f).

277
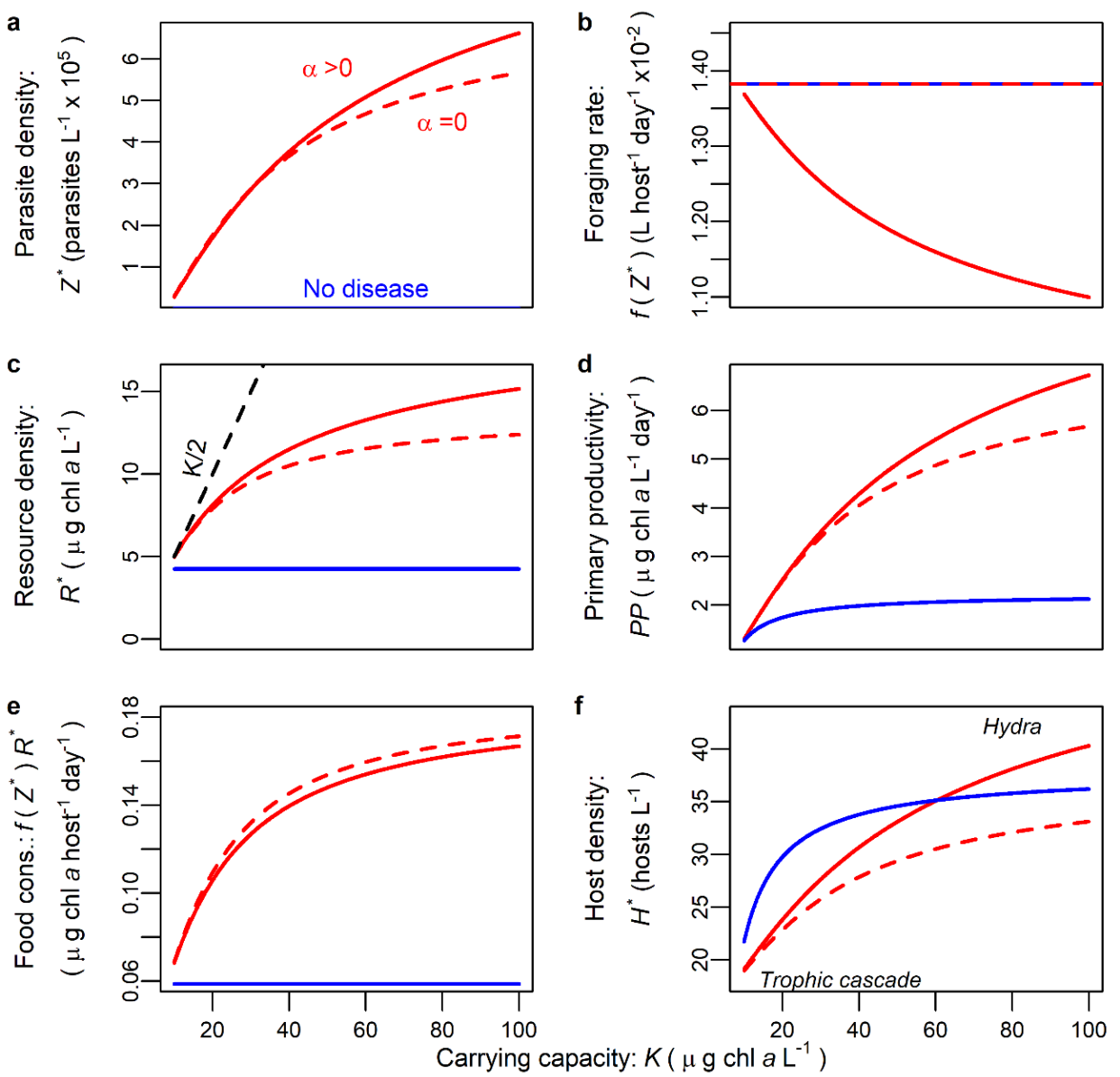

279 Figure 2. Foraging depression produces a hydra effect at high carrying capacity (see Fig. 3; eq.

2). Three cases shown: no-disease (blue, $Z$-), mortality-only (dashed red, $Z+; \alpha=0, v>0$ ), and foraging depression (solid red, $Z+; \alpha>0, v>0$ ). (a) Higher carrying capacity of the resource $(K)$ 
282 increases propagule density $\left(Z^{*}\right)$, dropping (b) foraging rate if $\alpha>0$. (c) Parasites release

283 resources $\left(R^{*}{ }^{+}>R^{*}{ }_{\text {-- }}\right) \cdot R^{*}{ }_{\mathrm{Z}}$ is closer to $K / 2$ (dashed black) with foraging depression. (d) Hence,

284 primary productivity $(P P)$ is highest when $\alpha>0$ and lowest without disease. (e) Food

285 consumption, $\left[F C=f\left(Z^{*}\right) R^{*}\right]$, is higher without foraging depression. (f) Given these responses of

$286 P P$ and $F C$, epidemics cause trophic cascades $\left(H^{*} Z_{-}>H^{*} Z^{+}\right.$always $)$without foraging depression

287 but can cause hydra effects when $\alpha>0$ at high $K$. (Parameters follow Table 1 ).

This framework predicts how carrying capacity $(K)$, susceptibility $(u)$, and foraging depression $(\alpha)$ jointly determine the range from strong cascades to hydra effects during epidemics. Although foraging rate drops with $\alpha$ and this releases resources, $K$ has a stronger 292 effect on resource release [indexed via ratio $\log _{10}\left(R^{*} Z^{+} / R^{*} Z_{-}\right)$, eq. $2 \mathrm{a}$, b; as parameterized, the contours are fairly vertical in Fig. 3a]. That resource release can then increase primary

294 productivity. Foraging depression (high $\alpha$ ) also decreases food consumption per host. Hence, the 295 host ratio $\left(H^{*}{ }_{Z+} / H^{*} Z_{-}\right)$increases with higher $K$ (due to enhanced $P P$ ) and higher $\alpha$ (via lower FC), 296 crossing from trophic cascade into hydra effect (past the black line $\left[\log _{10}\right.$ ratio $\left.=0\right]$ in Fig. $3 b$ ).

297 That hydra effect becomes less likely when hosts experience higher mean per capita mortality.

298 Mean mortality, $d+v p$, increases when susceptibility (higher $u$ ) raises prevalence (higher $p$ ).

299 Higher $u$ (hence higher mortality) releases resources strongly (larger resource ratios with higher

$300 \alpha$ but especially higher $u$; Fig. 3c), boosting primary production. However, higher $u$ also

301 increases food consumption per host. Therefore, despite larger resource release, higher

302 susceptibility makes hydra effects less likely (i.e., the region of host ratios sitting above the black

303 line shrinks with higher $u$; Fig. 3d; see Fig. S2 for an exception at low $u$ and high $\alpha$ ). Similarly, if 304 parasites exert higher pathogen-induced mortality, $v$, hydra effects become less likely (except at 
higher $\alpha$ and low $v$; see Fig. S3). Therefore, hydra effects arise at higher $K$ and when hosts mortality.
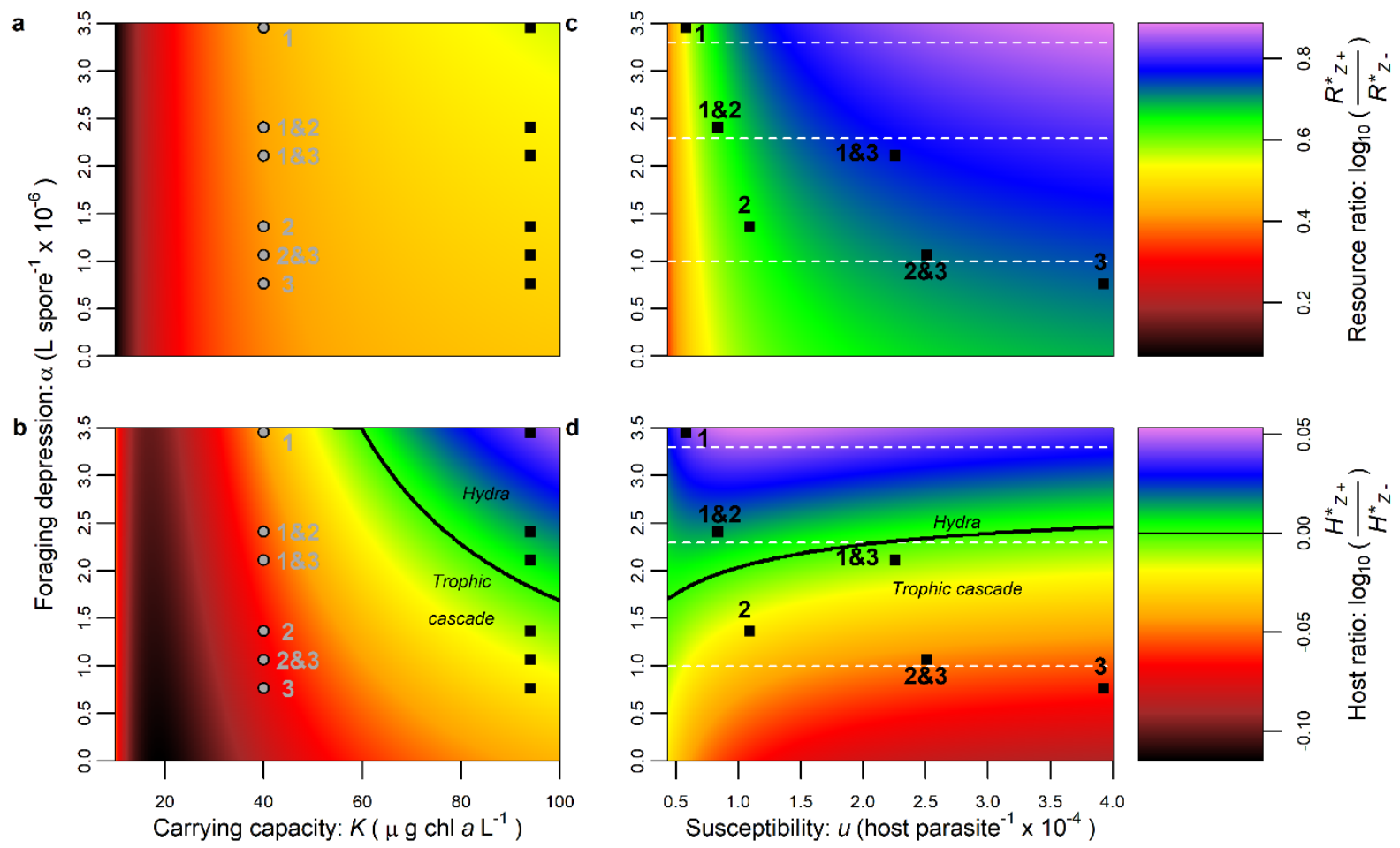

Figure 3. Combinations of carrying capacity (K, left column) or susceptibility (u, right) with

foraging depression ( $\alpha$ ) create cascades or hydra effects. Points indicate traits of numbered

312 genotype at low (light grey) or high (black) nutrient supply (note this comparison is qualitative).

313 Colors indicate $\log _{10}$ density ratios of resource (top row: $\log _{10}\left[R^{*} Z^{+} / R^{*} Z\right]$ ) or hosts (bottom:

$\left.314 \log _{10}\left[H^{*} Z^{+} / H^{*} Z-\right]\right)$. Black curves note host ratio $=0$ (above: hydra effects). $K$ - $\alpha$ space:

315 (intermediate $u=2.22 \times 10^{-4}$ ) (a) Resource ratio increases with $\alpha$ but especially $K$. (b) Both $K$ 316 and $\alpha$ jointly increase host ratio, yielding a hydra effect at high enough $K-\alpha$ (see Fig. 2 f). $u-\alpha$

317 parameter space: (high $K=94.3$ ) Slices at dotted white lines shown in Fig. S2. (c) Higher $u$ and 
$\alpha$ both increase resource ratio. (d) Higher susceptibility $(u)$ increases parasite-driven mortality, reducing the region of hydra effects in $u-\alpha$ space. (Parameters follow Table 1).

starting average traits $(u$ and $\alpha$ ) of mixed-genotype treatments are useful estimates for this qualitative prediction because the population is bounded within the traits of the two genotypes.

More specifically, trait values (see Figure 3) indicate where genotype treatments should fall along the spectrum from strong cascades to hydra effects. These patterns calculated from equilibria are qualitatively similar when the system is not yet in equilibrium in simulations or mesocosms (see Figs 4, S4, S5, S6). Similarly, the presence of two host clones does not alter the predicted impact of host traits (Figs S4, S6). Therefore, the model predicts patterns linking traits to the cascade-hydra effect spectrum for the experiment to test.

\section{(d) Mesocosm test of model predictions}

Most genotype treatments produced trophic cascades (released resources and suppressed host density). In populations with disease, higher susceptibility $(u)$ increased prevalence of fungal infection (found from beta regression; $\mathrm{P}=0.0067$; see Fig. S1a and Appendix: section 2d). Higher nutrients also increased prevalence of disease $(\mathrm{P}=0.0198)$, as predicted (see Fig.

S1a). Relative to disease-free populations, disease released algal resources overall $(\mathrm{P}=0.00126)$ and suppressed host density ( $\mathrm{P}=0.00137$; see Table $\mathrm{S} 1$ for more model-data comparison).

Susceptibility increased the strength of these trophic cascades, as predicted. Treatments with higher susceptibility displayed stronger release of algal resources (positive $u \mathrm{x} Z+$ interaction, $\mathrm{P}$ $=0.0277$; see Figs S5d-f, S6d-f; increasing resource ratio in Fig. 5a). Susceptibility also 
341 strengthened host suppression (negative $u \mathrm{x} Z+$ interaction, $\mathrm{P}<0.001$; Figs 4d-f, S4d-f;

342 decreasing host ratio in Fig. 5b). These results match predictions for parasite-driven, density-

343 mediated trophic cascades (see Figs 3c, d, 4a-c, S4a-c, S5a-c, S6a-c).

344 However, two sets of treatments displayed a hydra effect. At high nutrient supply, host

345 density was higher with disease than without it for genotype 1 alone (strong hydra: 79\% higher

346 with disease, $\mathrm{P}=0.00748$; Figs $3 \mathrm{~d}$, 4a, and square labeled ' 1 ' in $5 \mathrm{~b}$ ) and for the mixture of

347 genotypes $1 \& 2$ (weaker hydra: $34 \%$ higher, $\mathrm{P}=0.0201$; Figs $\mathrm{S} 4 \mathrm{a}$ and square ' $1 \& 2$ ' in 5 b).

348 Since genotype 1 has low susceptibility $(u)$ and high coefficient of foraging depression $(\alpha$; Fig.

349 1), these results follow the model prediction for hydra effects at high $K$, lower $u$, and lower $\alpha$

350 (see Figs 3b, d, 4a, S4a). 

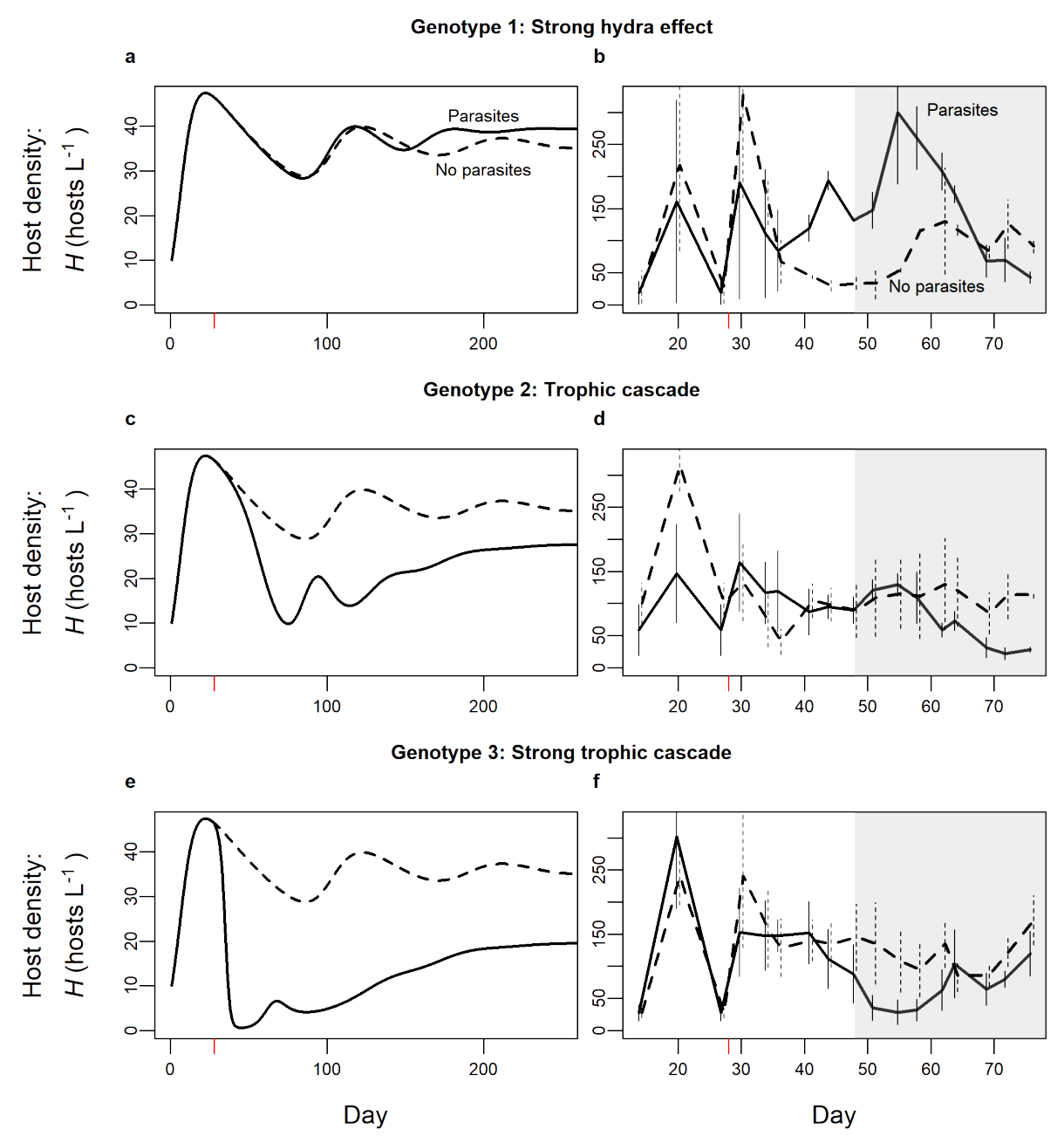

353 Figure 4. Simulated and experimental time series at high nutrients ( $\mathrm{K}=94.3$ in simulations or

$35450 \mu \mathrm{g} \mathrm{L}^{-1} \mathrm{P}$ in mesocosms) produce a spectrum ranging from hydra effects to trophic cascades.

355 In both simulations and the experiment, hosts and parasites are added on days 0 and 28 (red tick 356 mark), respectively. (a) With genotype 1's traits, the hydra effect emerges given sufficient time 357 as host density with parasites (solid) becomes higher than without (dashed). (b) Mesocosms of 358 genotype 1 experienced a hydra effect [mean density across replicates with parasites (solid) or 359 without (dashed), plotted at each time point; bars are standard error at each time point]. (c) With 360 genotype 2's traits, a trophic cascade (host suppression and resource release [Fig. S5]) occurs in 361 simulations and (d) the mesocosm. (e-f) This cascade is larger for genotype 3 (the most 
362 susceptible). (Parameters follow Table 1). For analyses, average mesocosm density was taken

363 from day 48 to 76 (gray region, see Methods for mesocosm). Experimental time series shifted

364 slightly horizontally for clarity. Compare simulations to Fig. 3's equilibrium outcomes and

365 mesocosm time series to Fig. 5's mesocosm averages.

a

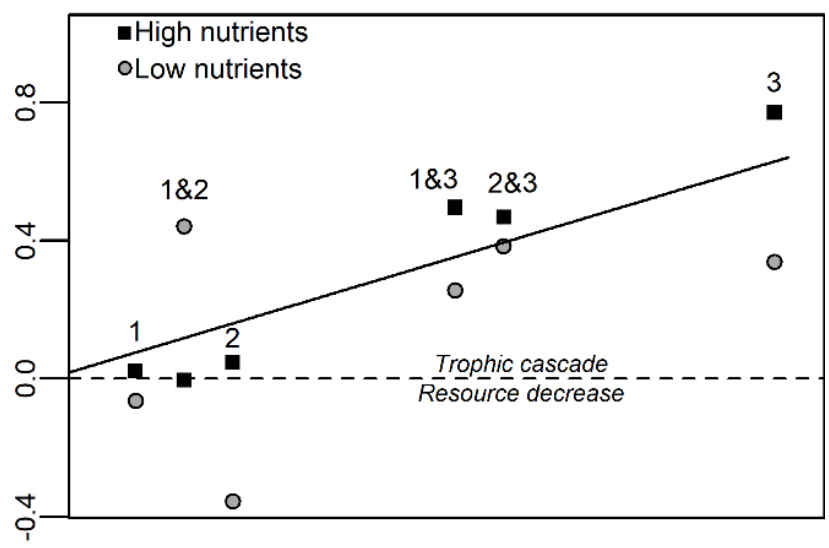

b

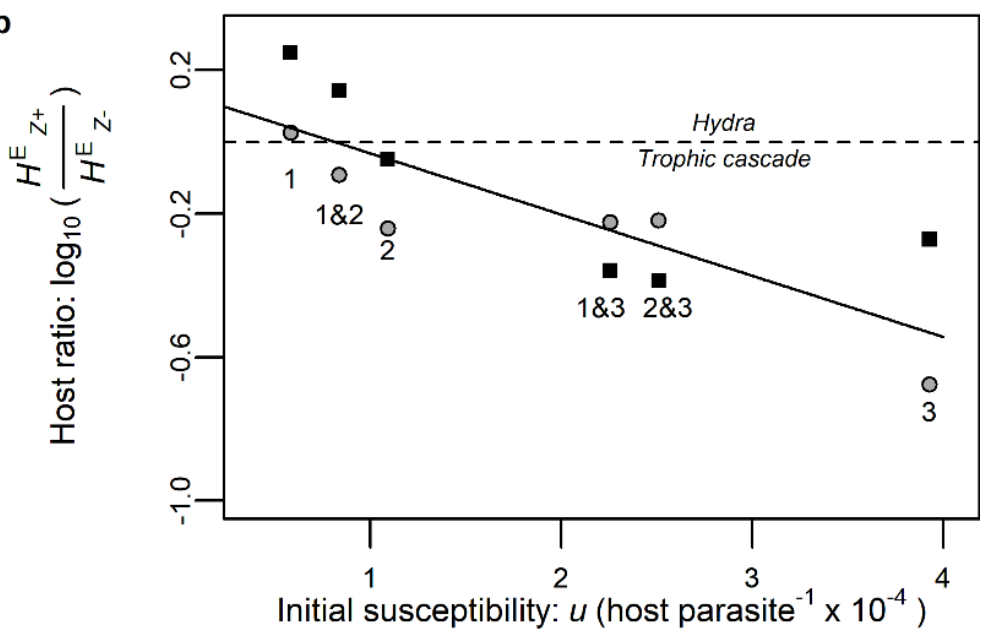

Figure 5. In experimental epidemics, parasite drove cascades or hydra effects depending on susceptibility (u), foraging depression ( $\alpha$ ), and nutrient supply $(K)$. Each point represents the

371 average resource or host ratio. Algal resources: $R^{E}$ (E for 'experiment'). (a) Higher susceptibility

372 amplifies resource release (increasing $\log _{10}$ ratio of resources). (Error bars for ratios not shown: 
373 they are non-trivial to compute). Plankton hosts: $H^{E}$. (b) Higher susceptibility amplifies trophic

374 cascades (stronger host suppression; $\log _{10}$ host ratios become more negative with $u$ ). However,

375 two genotype treatments with low susceptibility and strong foraging depression (1 and $1 \& 2$

376 together) displayed disease-driven hydra effects $\left(\log _{10}\right.$ host ratios $\left.>0\right)$ at high nutrient supply.

377

\section{4. Discussion}

Our model qualitatively anticipated the strength of parasite-driven trophic cascades and hydra effects in a mesocosm experiment. Higher susceptibility of host genotypes increased prevalence of fungal infection, suppressed density of zooplankton hosts, and released algal resources. This result echoes how higher attack rate on prey strengthens predator-driven trophic cascades. However, at high nutrient supply, the least susceptible genotype experienced a partially because these treatments had lower susceptibility, hence lower per capita mortality rate. Vitally, these genotype treatments also showed strongest depression of foraging rate when encountering parasite propagules. Our model predicted that this combination of lower susceptibility and higher foraging depression could make parasites increase resource production (especially at high nutrients) more than food consumption during epidemics. Hence, this traitenvironment combination predicted the strength of parasite-driven trophic cascades but also the appearance of hydra effects.

Susceptibility strengthened trophic cascades because it increased disease prevalence,

393 hence parasite-driven mortality of hosts. We tested this prediction by manipulating susceptibility

394 via a range of clonal genotypes of host populations. The susceptible genotypes, alone and in

395 combination with each other, produced stronger parasite-driven trophic cascades (i.e., stronger 
host suppression and resource release). Parasite-driven trophic cascades are a well-established

397 phenomenon (Buck \& Ripple 2017); our model-experiment match adds this emerging area as the

398 first to predict the strength of parasite-driven trophic cascades. It also demonstrates the power of

399 the analogy to predator-driven cascades (Hall et al. 2008; Raffel, Martin \& Rohr 2008 and see

400 Appendix: section 1a for more details). Like in the disease case, higher mortality inflicted by

401 predators should lead to stronger cascades. Hence, predator-driven cascades should be stronger

402 when predators capture prey more quickly (analogous to higher transmission rate and related

403 higher susceptibility; Shurin \& Seabloom 2005). Additionally, factors that enhance attack rate -

404

405

406

407

408

409

410

411

412

413

414

415

416

417 equal, this higher production could support higher host density. However, increased mortality

418 increases per-capita food consumption of hosts. If parasites only kill hosts but do not change 
419 foraging rate, food consumption always increases more than resource production (in our model).

420 These parasites can only cause trophic cascades (see Buck \& Ripple 2017 for many examples).

421 In contrast, a hydra effect can arise when parasites depress foraging rate of hosts. Foraging

422 depression, a trait-mediated effect, arises commonly in this host-parasite system (Hite et al.

423 2017; Strauss et al. 2019) and others (Hite, Pfenning \& Cressler 2020) and in predator-prey

424 systems (Morgan 1988; Laundré, Hernández \& Ripple 2010). Here, foraging depression enables

425 production to increase more than per capita consumption of resources during epidemics. In such

426 circumstances, foraging depression can create hydra effects.

Both the dynamical model and the experiment anticipate conditions needed for a parasite-

428 driven hydra effect. Hosts with low susceptibility (low $u$ ) suffer less infection and infection-

429 induced mortality (pathogen-induced mortality). Lower mortality coupled with stronger

430 depression of foraging rate (higher $\alpha$ ) can cause resource release without huge increases in food

431 consumption. Furthermore, resource release can elevate primary productivity more at higher

432 carrying capacity (when $R^{*}$ of the host without disease is less than $K / 2$ ). The experiment verified

433 these predictions; hydra effects arose for hosts having both low susceptibility and strong foraging

434 depression in environments with high supply of nutrients (high $K$ ). This example with parasites

435 adds to a few other examples of hydra effects caused by mortality fixed by the experiment and

436 falls under a "prudent resource exploitation" mechanism (Abrams 2009; Schröder, van Leeuwen

437 \& Cameron 2014). Previously, others have shown how parasites can increase biomass

438 (Ohlberger et al. 2011; Preston \& Sauer 2020) or both body mass and survival for a specific life

439 stage of hosts (Washburn \& Mercer 1991). Total host density also increased in large fungal

440 epidemics in this host in lake populations (Penczykowski et al. 2020). Here, in a controlled

441 experiment, parasites increased total host density. That outcome emerged due to dynamical 
442 feedbacks from interacting hosts, resources, and parasites over multiple generations.

443

444

\section{5}

446

447

448

449

450

451

\section{2}

Given its emergence here, how can we anticipate parasite-driven hydra effects in other systems? What factors might constrain or amplify their possibility? First, the relationship between susceptibility $(u)$ and foraging depression $(\alpha)$ traits may make hydra effects more or less likely. In the set of genotypes here, susceptibility and foraging depression were negatively correlated, yielding strong trophic cascades (high $u$, low $\alpha$; lower right Fig. 3d) or strong hydra effects (low $u$, high $\alpha$; upper left Fig. 3d). For another set of genotypes of this host, these traits did not correlate negatively (Strauss et al. 2019). A positive correlation might reduce the probability of strong cascades or strong hydra effects. Second, hosts may evolve weaker or stronger foraging depression as selection weighs lowered fecundity against lowered exposure to parasites. Third, hydra effects should be somewhat less likely when parasites impose more harm to host fitness. For systems where foraging depression does not reduce parasite exposure, exposure and parasite-induced mortality would remain high, making a hydra effect somewhat less likely. Similarly, parasites imposing high virulence on mortality and on fecundity are less likely to drive hydra effects. Castrators, for example, should more likely cause cascades (see Appendix: section 1b). Future work could clarify the importance of each of these factors for hydra effects in this and other systems.

Here, using models and experiments, we delineate when parasites cause trophic cascades or hydra effects. Parasite-driven trophic cascades have emerged in various systems. Here, we develop and test a trait-based model framework experimentally. Yet, the parasite-mediated hydra effect that arose reveals an even newer possibility: parasites that kill hosts could increase host density if they depress foraging. This finding seems baffling until one embraces feedbacks between hosts and resources. Now disease ecologists can ask: are disease-mediated hydra effects 
465 rare? Or could they arise more commonly - if we just know where and when to look for them?

466 Here, the mathematical model guides us. First, parasites must not increase host mortality too

467 greatly. Second, hosts must depress their foraging rate in the presence of parasites (a trait-

468 mediated effect). Third, higher resource density must increase primary productivity. If resources

469 cannot respond dynamically to host density or if productivity does not increase with higher

470 resources, hydra effects cannot occur via this prudent resource exploitation mechanism.

471 Nonetheless, we show how these three factors (mortality, foraging depression, productivity)

472 govern the range from strong parasite-driven trophic cascades to hydra effects.

473

474 Acknowledgements: O. Schmidt and J. Obergfell provided assistance with the experiments. C.

475 Lively, F. Bashey-Visser, M. Wade, A. Ramesh, and T. Deblieux provided valuable feedback on

476 the manuscript. This work was supported by NSF DEB 1353749, 1655656, and an NSF GRFP

4771342962 to J. Walsman.

478 Author contributions: JW and SH designed the mesocosm experiment, AS and SH designed the

479 trait measurements, AS performed the trait measurements, JW performed the mesocosm

480 experiment, mathematical modeling, data analysis, and wrote the first draft of the manuscript

481 while all authors contributed substantially to revisions.

\section{$\underline{\text { References }}$}

483 Abrams, P.A. (2009) When does greater mortality increase population size? The long history and diverse mechanisms underlying the hydra effect. Ecology Letters, 12, 462-474.

485 Beckerman, A.P., Uriarte, M. \& Schmitz, O.J. (1997) Experimental evidence for a behavior486 mediated trophic cascade in a terrestrial food chain. Proceedings of the National Academy of Sciences, 94, 10735-10738. 
488

489

490

491

492

493

494

495

496

497

498

499

500

501

502

503

504

505

506

507

508

509

510

Boivin, G., Hance, T. \& Brodeur, J. (2012) Aphid parasitoids in biological control. Canadian Journal of Plant Science, 92, 1-12.

Borer, E.T., Seabloom, E.W., Shurin, J.B., Anderson, K.E., Blanchette, C.A., Broitman, B., Cooper, S.D. \& Halpern, B.S. (2005) What determines the strength of a trophic cascade? Ecology, 86, 528-537.

Buck, J., Weinstein, S. \& Young, H. (2018) Ecological and evolutionary consequences of parasite avoidance. Trends in ecology \& evolution, 33, 619-632.

Buck, J.C. \& Ripple, W.J. (2017) Infectious agents trigger trophic cascades. Trends in ecology \& evolution, 32, 681-694.

Cleaveland, S., Laurenson, M. \& Taylor, L. (2001) Diseases of humans and their domestic mammals: pathogen characteristics, host range and the risk of emergence. Philosophical Transactions of the Royal Society of London. Series B: Biological Sciences, 356, 991999.

Cooper, J., Crawford, R.J., De Villiers, M.S., Dyer, B.M., Hofmeyr, G.G. \& Jonker, A. (2009) Disease outbreaks among penguins at sub-Antarctic Marion Island: a conservation concern. Marine Ornithology, 37, 193-196.

Daszak, P., Cunningham, A.A. \& Hyatt, A.D. (2000) Emerging infectious diseases of wildlife-threats to biodiversity and human health. science, 287, 443-449.

Dobson, A., Lafferty, K.D., Kuris, A.M., Hechinger, R.F. \& Jetz, W. (2008) Homage to Linnaeus: how many parasites? How many hosts? Proceedings of the National Academy of Sciences, 105, 11482-11489.

Dwyer, G. \& Elkinton, J.S. (1993) Using simple models to predict virus epizootics in gypsy moth populations. Journal of Animal Ecology, 1-11. 
511

512

513

514

515

516

517

518

519

520

521

522

523

524

525

526

527

528

529

530

531

532

533

Ebert, D. (2005) Ecology, epidemiology, and evolution of parasitism in Daphnia. National Library of Medicine (US), National Center for Biotechnology Information, Bethesda, MD, United States.

Ebert, D., Lipsitch, M. \& Mangin, K.L. (2000) The effect of parasites on host population density and extinction: Experimental epidemiology with Daphnia and six microparasites. American Naturalist, 156, 459-477.

Efron, B. \& Tibshirani, R.J. (1993) An Introduction to the Bootstrap. Chapman \& Hall, New York.

Fourqurean, J.W., Manuel, S., Coates, K.A., Kenworthy, W.J. \& Smith, S.R. (2010) Effects of excluding sea turtle herbivores from a seagrass bed: overgrazing may have led to loss of seagrass meadows in Bermuda. Marine Ecology Progress Series, 419, 223-232.

Fry, W.E. \& Goodwin, S.B. (1997) Resurgence of the Irish potato famine fungus. BioScience, 47, 363-371.

Hall, S.R., Becker, C.R., Duffy, M.A. \& Cáceres, C.E. (2011) Epidemic size determines population-level effects of fungal parasites on Daphnia hosts. Oecologia, 166, 833-842.

Hall, S.R., Lafferty, K.D., Brown, J.H., Cáceres, C.E., Chase, J.M., Dobson, A.P., Holt, R.D., Jones, C.G., Randolph, S.E. \& Rohani, P. (2008) Is infectious disease just another type of predator-prey interaction. Infectious Disease Ecology: the effects of ecosystems on disease and of disease on ecosystems, 223-241.

Hedges, L.V., Gurevitch, J. \& Curtis, P.S. (1999) The meta-analysis of response ratios in experimental ecology. Ecology, 80, 1150-1156.

Hite, J.L., Penczykowski, R.M., Shocket, M.S., Griebel, K.A., Strauss, A.T., Duffy, M.A., Cáceres, C.E. \& Hall, S.R. (2017) Allocation, not male resistance, increases male 
frequency during epidemics: a case study in facultatively sexual hosts. Ecology, 98, 2773-2783.

Hite, J.L., Pfenning, A.C. \& Cressler, C.E. (2020) Starving the enemy? Feeding behavior shapes host-parasite interactions. Trends in ecology \& evolution, 35, 68-80.

Hochachka, W.M. \& Dhondt, A.A. (2000) Density-dependent decline of host abundance resulting from a new infectious disease. Proceedings of the National Academy of Sciences, 97, 5303-5306.

Holdo, R.M., Sinclair, A.R., Dobson, A.P., Metzger, K.L., Bolker, B.M., Ritchie, M.E. \& Holt, R.D. (2009) A disease-mediated trophic cascade in the Serengeti and its implications for ecosystem C. PLoS Biol, 7, e1000210.

Horan, R.D. \& Fenichel, E.P. (2007) Economics and ecology of managing emerging infectious animal diseases. American journal of agricultural economics, 89, 1232-1238.

Jochum, M., Schneider, F.D., Crowe, T.P., Brose, U. \& O'Gorman, E.J. (2012) Climate-induced changes in bottom-up and top-down processes independently alter a marine ecosystem. Philosophical Transactions of the Royal Society B: Biological Sciences, 367, 2962-2970.

Katano, O., Natsumeda, T. \& Suguro, N. (2013) Diurnal bottom feeding of predator fish strengthens trophic cascades to benthic algae in experimental flow-through pools. Ecological Research, 28, 907-918.

Laundré, J.W., Hernández, L. \& Ripple, W.J. (2010) The landscape of fear: ecological implications of being afraid. The Open Ecology Journal, 3.

Matsuda, H. \& Abrams, P.A. (2004) Effects of predator prey interactions and adaptive change on sustainable yield. Canadian Journal of Fisheries and Aquatic Sciences, 61, 175-184. 
Morgan, M.J. (1988) The influence of hunger, shoal size and predator presence on foraging in bluntnose minnows. Animal Behaviour, 36, 1317-1322.

Ohlberger, J., Langangen, Ø., Edeline, E., Claessen, D., Winfield, I.J., Stenseth, N.C. \& Vøllestad, L.A. (2011) Stage-specific biomass overcompensation by juveniles in response to increased adult mortality in a wild fish population. Ecology, 92, 2175-2182.

Overholt, E.P., Hall, S.R., Williamson, C.E., Meikle, C.K., Duffy, M.A. \& Caceres, C.E. (2012) Solar radiation decreases parasitism in Daphnia. Ecology Letters, 15, 47-54.

Penczykowski, R.M., Hall, S.R., Shocket, M.S., Ochs, J.H., Lemanski, B.C., Sundar, H. \& Duffy, M.A. (2020) Virulent disease epidemics can increase host density by depressing foraging of hosts. bioRxiv.

Philpott, S.M., Maldonado, J., Vandermeer, J. \& Perfecto, I. (2004) Taking trophic cascades up a level: behaviorally-modified effects of phorid flies on ants and ant prey in coffee agroecosystems. Oikos, 105, 141-147.

Preston, D.L. \& Sauer, E.L. (2020) Infection pathology and competition mediate host biomass overcompensation from disease. Ecology, 101.

R Core Team (2019) R: A language and environment for statistical computing. R Foundation for Statistical Computing, Vienna, Austria.

Raffel, T.R., Martin, L.B. \& Rohr, J.R. (2008) Parasites as predators: unifying natural enemy ecology. Trends in ecology \& evolution, 23, 610-618.

Raveh, A., Kotler, B.P., Abramsky, Z. \& Krasnov, B.R. (2011) Driven to distraction: detecting the hidden costs of flea parasitism through foraging behaviour in gerbils. Ecology Letters, 14, 47-51. 
578 Roelke-Parker, M.E., Munson, L., Packer, C., Kock, R., Cleaveland, S., Carpenter, M., O'brien,

579 S.J., Pospischil, A., Hofmann-Lehmann, R. \& Lutz, H. (1996) A canine distemper virus epidemic in Serengeti lions (Panthera leo). Nature, 379, 441.

581

582

583

584

585

586

587

588

589

590

591

592

593

594

595

596

597

598

599

Schröder, A., van Leeuwen, A. \& Cameron, T.C. (2014) When less is more: positive populationlevel effects of mortality. Trends in ecology \& evolution, 29, 614-624.

Shurin, J.B. \& Seabloom, E.W. (2005) The strength of trophic cascades across ecosystems: predictions from allometry and energetics. Journal of Animal Ecology, 74, 1029-1038.

Simonis, J.L. (2013) Predator ontogeny determines trophic cascade strength in freshwater rock pools. Ecosphere, 4, 1-25.

Stewart Merrill, T.E., Hall, S.R., Merrill, L. \& Cáceres, C.E. (2019) Variation in immune defense shapes disease outcomes in laboratory and wild Daphnia. Integrative and Comparative Biology, 59, 1203-1219.

Strauss, A.T., Bowling, A.M., Duffy, M.A., Cáceres, C.E. \& Hall, S.R. (2018) Linking host traits, interactions with competitors and disease: mechanistic foundations for disease dilution. Functional Ecology, 32, 1271-1279.

Strauss, A.T., Civitello, D.J., Cáceres, C.E. \& Hall, S.R. (2015) Success, failure and ambiguity of the dilution effect among competitors. Ecology Letters, 18, 916-926.

Strauss, A.T., Hite, J.L., Civitello, D.J., Shocket, M.S., Cáceres, C.E. \& Hall, S.R. (2019) Genotypic variation in parasite avoidance behaviour and other mechanistic, nonlinear components of transmission. Proceedings of the Royal Society B, 286, 20192164.

Thrall, P. \& Burdon, J. (2000) Effect of resistance variation in a natural plant host-pathogen metapopulation on disease dynamics. Plant Pathology, 49, 767-773. 
600 Vredenburg, V.T., Knapp, R.A., Tunstall, T.S. \& Briggs, C.J. (2010) Dynamics of an emerging 601 disease drive large-scale amphibian population extinctions. Proceedings of the National Academy of Sciences, 107, 9689-9694.

603 Vucic-Pestic, O., Ehnes, R.B., Rall, B.C. \& Brose, U. (2011) Warming up the system: higher

604 predator feeding rates but lower energetic efficiencies. Global Change Biology, 17, 1301-

605 1310.

606 Washburn, J.O. \& Mercer, D.R. (1991) Regulatory role of parasites: impact on host population shifts with resource availability. science, 253, 185-188.

608 Wood, C.L., Byers, J.E., Cottingham, K.L., Altman, I., Donahue, M.J. \& Blakeslee, A.M. (2007)

609 Parasites alter community structure. Proceedings of the National Academy of Sciences,

610 104, 9335-9339.

611 